\title{
Experimental Evaluation of Bipolar Surface Potential with Corona Charging for Electret Activation
}

\author{
Koki Yamamoto, ${ }^{1 *}$ Takayuki Fujita, ${ }^{2}$ Adrien Badel, ${ }^{3}$ \\ Fabien Formosa, ${ }^{3}$ Kensuke Kanda, ${ }^{2}$ and Kazusuke Maenaka ${ }^{2}$ \\ ${ }^{1}$ Fundamental Technologies Research Department, Technologies and R\&D Division, Ebara Corporation, \\ 4-2-1 Honfujisawa, Fujisawa, Kanagawa 251-8502, Japan \\ ${ }^{2}$ Graduate School of Engineering, University of Hyogo, 2167 Shosha, Himeji, Hyogo 671-2280, Japan \\ ${ }^{3}$ SYMME Laboratory, Université Savoie Mont Blanc, 11 Chemin de bellevue, Annecy-le-vieux 74940, France
}

(Received April 8, 2020; accepted June 15, 2020)

Keywords: vibration energy harvester, MEMS, bipolar charging, electret, experimental evaluation

In this work, we focus on the experimental evaluation of the bipolar surface potential in a MEMS electret vibration energy harvester. Bipolar charging increases the absolute electrical energy of an electret when the electrostatic force is kept low. Measuring the practical surface potential is necessary for the design and optimization of the harvester. However, it is difficult to estimate the potential after assembly because the typical approach using surface potentiometers is not applicable. In this work, an evaluation method for bipolar charging in conjunction with an external voltage source was developed. To evaluate the proposed method, a typical MEMS electret vibration energy harvester was fabricated. In the experiment, the harvested voltage and displacement at a low acceleration of $0.2 \mathrm{mg}$ (gravitational acceleration, $g=9.81 \mathrm{~m} / \mathrm{s}^{2}$ ) were measured. Since the proposed method is applied at harvesting, the harvested voltage can be changed with an external voltage. The proposed method shows that factors including the relative responses of the harvested voltage can be estimated using the practical potential. In this work, the surface potential in the electret was explicitly considered in the harvesting experiment using a practical bipolar MEMS electret-based vibration energy harvester and an external voltage source.

\section{Introduction}

Over the past decade, applications of Internet of Things (IoT) technology have been strongly anticipated in consumer activities, specifically industrial applications that reduce personal expenses, develop smart factories, and reduce risk by accident prevention. ${ }^{(1-3)}$ This technology will be a novel strategy to drive the fourth industrial revolution in manufacturing [Industry $4.0^{(4)}$ ]. A semipermanent battery is essential for this technology to expand its industrial applications. Autonomous batteries, which collect environmental energy and convert it into electrical energy, are one of the leading candidates that dispel the concerns about the lifetime of primary batteries. Typical energy harvesting (EH) techniques to capture environmental energies, such as solar, vibrational, thermal, and radio frequency energies, into mesoscale devices have been "Corresponding author: e-mail: yamamoto.koki@ebara.com https://oi.org/10.18494/SAM.2020.2901 
studied. ${ }^{(5)}$ In conjunction with MEMS technology, miniaturized electrical elements can be used to increase the harvesting power density. The vibrating energy harvester (VEH) structure is easy to apply to MEMS because its mechanical and electrical components can be completely processed by micromachining. The VEH system is classified into the transducer type, which is piezoelectric, ${ }^{(6,7)}$ electromagnetic, ${ }^{(8)}$ or electrostatic. ${ }^{(9)}$ The electrostatic VEH (ES-VEH) has attracted much attention for its application to low-frequency vibration sources ${ }^{(10)}$ and is characterized by its small volume and high power density compared with other transducers. We have proposed a bipolar charging ES-VEH structure for industrial applications. ${ }^{(11)}$ In this work, the ES-VEH device is based on an electret material that can keep the surface potential within itself.

\section{Electret Material Activation of MEMS ES-VEH Structure}

ES-VEHs must retain the ions stored to drive itself. The harvester structure, which combines a variable capacitor and an electrical energy source, can generate output power. Most of the ES-VEHs are based on electrets, ${ }^{(12)}$ whereas others generate electrical energy through an external power source. ${ }^{(13)}$ We combine an electret-based ES structure with a simple structure without an external driving circuit. To activate the electret material, charging is necessary. The corona discharge method is one of the typical and simplest methods known to realize an electret-based ES-VEH. ${ }^{(14)}$ In this study, the corona discharge method is used to selectively charge the bipolar charging structure. A typical MEMS ES-VEH structure often uses a monopolar charger that can only keep negative or positive ions inside. ${ }^{(15)}$ This method is the traditional way of providing an ES transducer in a component. However, owing to the pull-in effect of both electrodes, the electrodes may suddenly become fixed owing to the electrostatic force between them, which can prevent them from storing a large potential. The ES-VEH structure is also necessary to increase the potential for a large output, which is proportionally squared as a variable parameter. Bipolar charging reduces the electrostatic force in the presence of an equivalent monopolar charging. The applied potential is set as a bias voltage at which the surface potential can be determined. ${ }^{(16)}$ To optimize the ES structure, it is important to estimate the practical surface potential and capacitance. In this work, we focus on evaluating the surface potential after assembly. Since the ES-VEH is already monolithically packaged, it is difficult to directly measure the surface potential of bipolar charging after its assembly. Typically, the surface potential is measured using a noncontact voltage potentiometer for monopolar charging. ${ }^{(17)}$ Its ideal operation for measuring the surface potential before assembly is shown in Fig. 1(a). The electrodes for monopolar charging are shown and consist of a potentiometer and a noncontact probe. This method can easily be applied to large-width patterns, and if the electrode pattern is much smaller than the resolution of the probe, the potential is not correct, so the measured voltage reaches $1 / 2 V_{c p}$, which is approximately half the amplitude of the actual surface potential. Figure 1(b) shows a schematic diagram of bipolar charging. The noncontact method could not be applied to bipolar charging because the average voltage amplitude would be zero in the bipolar ES-VEH owing to electrical symmetry, so the method does not include any effects of charges. Thus, a novel strategy is proposed in this study; it combines an external voltage source that can indicate the various amplitude responses of the harvesting voltage with 

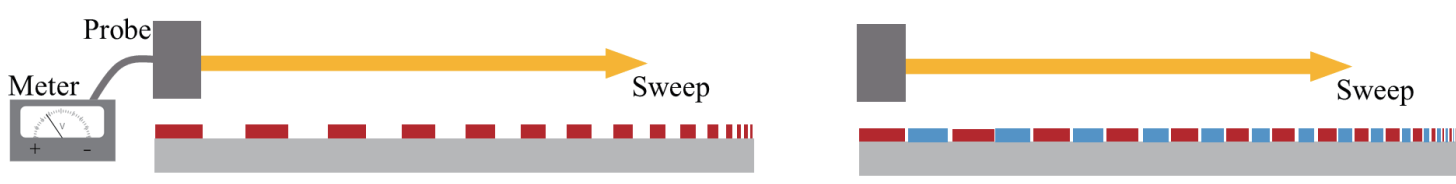

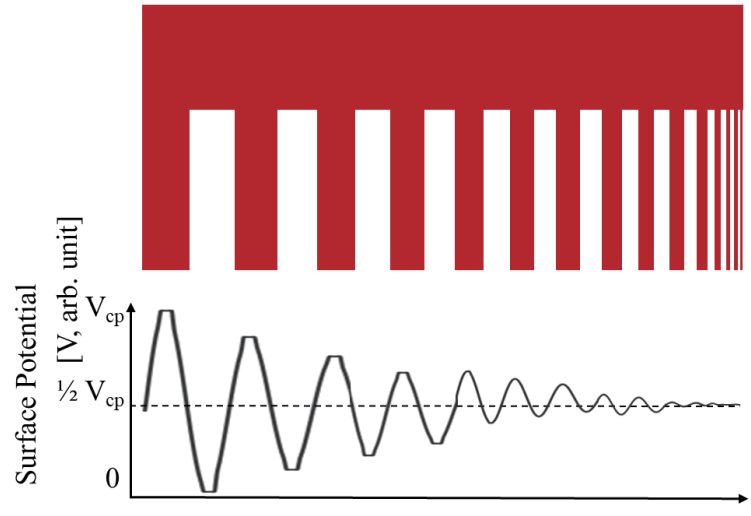

(a)
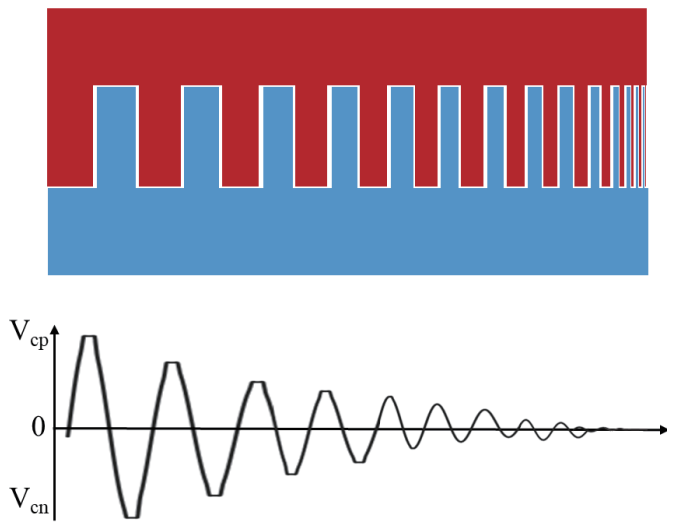

(b)

Fig. 1. (Color online) Schematic diagram of surface potential measurement for electret charge model. ${ }^{(17)}$ (a) Monopolar charge. (b) Bipolar charge.

the increase and decrease in electrical bias using voltage sources for measuring the potential. This method can be applied to an assembled bipolar ES-VEH for evaluating the charge condition even when the device is operated in the harvesting mode.

\section{Bipolar Electret-based MEMS VEH Structure}

Figure 2 shows a schematic diagram of the bipolar charging electret-based ES-VEH structure and the equivalent circuit. ${ }^{(18,19)}$ The cross section of the bipolar charging electret-based ESVEH is shown in Fig. 2(a). The simulation model consists of a supported substrate, base electrodes $\left(\mathrm{BE}_{1}\right.$ and $\left.\mathrm{BE}_{2}\right)$, electrets, and variable capacitors. Four of the variable capacitors are air-gap-dependent, $C_{n 1}, C_{n 2}, C_{p 1}$, and $C_{p 2}$, and can generate harvesting energy, and the parasitic capacitor in the counter electrode is denoted as $C_{12}$. The other parasitic capacitors between CEs and BEs are written as $C_{p a r_{-} n}$ and $C_{\text {par }} p$. The positive and negative surface potentials are placed on the electret film on the base electrode, which is located about $1 \mu \mathrm{m}$ from the top surface. ${ }^{(20)}$ The capacitors of the electret material are denoted as $C_{c y t_{-} n}$ and $C_{c y t} p$. The counter electrodes $\left(\mathrm{CE}_{1}\right.$ and $\left.\mathrm{CE}_{2}\right)$ are electrically isolated and can be driven separately. This structure generates electrical energy supplied by two types of variable capacitance $\left(C_{n 1,2}\right.$ and $\left.C_{p 1,2}\right)$ that depend on the seismic mass and the relative displacement of the opposing electrode. Current is generated through the CEs and BEs. The practical device consists of 50 pairs of comb structures. In the structure, the load resistances are connected between the CEs and the ground so that the energy is taken from two electrically separated wires.

Figure 2(b) shows a diagram illustrating the equivalent circuit of the harvester described with respect to Fig. 2(a). The surface potentials consisting of negative and positive biases for achieving the bipolar potential are rewritten as $V_{c n}$ and $V_{c p}$, respectively. 


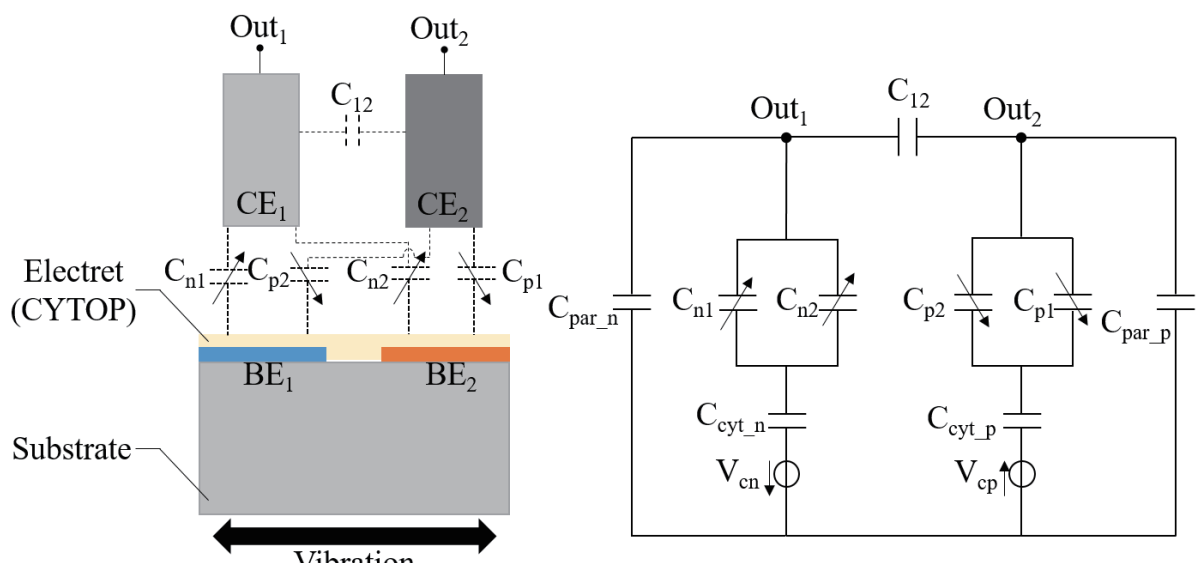

Vibration

(a) (b)

Fig. 2. (Color online) Bipolar charged electret-based ES-VEH structure: (a) cross-sectional drawing and (b) equivalent circuit.

\section{Fabrication of MEMS ES-VEH}

The fabrication process that realizes the harvester structure using silicon MEMS is based on a previous work. $^{(19)}$ The prototype consists of three layers: a counter electrode, a transducer, and a cover glass. The assembled prototype is shown in Fig. 3. The spring and transducer components include a linear resonator based on an electret material (CYTOP CTL-809M, AGC Corporation), a seismic mass, and a transducer. The CE components are based on lowresistance silicon wafers and aluminum electrodes connected to electrical load. As shown in Fig. 3(a), the cover glass wafer is pyrex prepared by glass wet etching with hydrofluoric acid to fabricate a spacer about $30 \mu \mathrm{m}$ higher than the center of the device. After fabrication, the chips are assembled with epoxy resin (KMPR1025, MicroChem) and heating. An overview of the completed device is shown in Fig. 3(b). The overall size is $12 \times 12 \times 1.2 \mathrm{~mm}^{3}$. The surface potential for activating the electret material is set to $\pm 150 \mathrm{~V}$. The device is connected to the substrate and receives electrical energy through the wire by using an ultrasonic wire bonder. The electrical energy is output through a variable capacitor for load resistance. The mechanical resonance frequency is chosen to be $300 \mathrm{~Hz}$, but the practical resonance frequency has been measured to be $313 \mathrm{~Hz}$ because of the fabrication accuracy. The electrode width is chosen to be $75 \mu \mathrm{m}$, which was optimized in a previous work. For variable capacitor designs, the air gap should be set to $30 \mu \mathrm{m}$. The practical variable capacitances $C_{n 1}, C_{n 2}, C_{p 1}, C_{p 2}$, and $C_{12}$ measured after charging are 19.3, 28.2, 28.3, 27.6, and $53.1 \mathrm{pF}$, respectively, including initial position fabrication errors and position errors. The capacitance $C_{n 1}$ is shown to be smaller than the other capacitances, because it includes a small parasitic resistance. The capacitances of the CYTOP material, $C_{c y t \_n}$ and $C_{c y t} p$, were estimated to be both $185 \mathrm{pF}$, with a thickness of $3 \mu \mathrm{m}$ and a relative permittivity of 2.1 . 


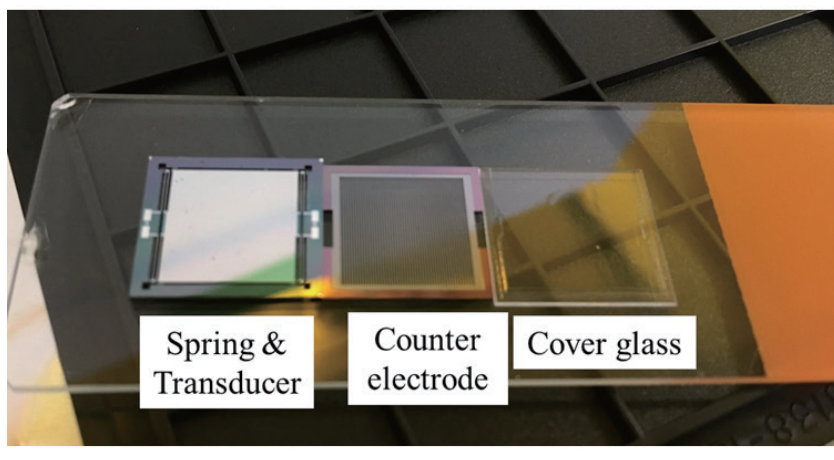

(a)

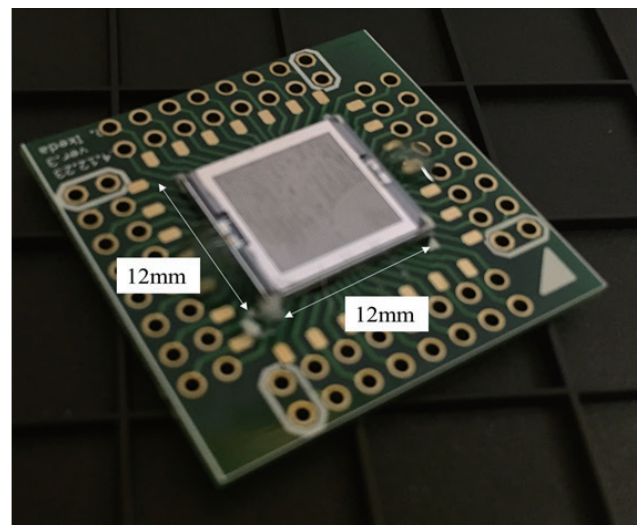

(b)

Fig. 3. (Color online) Assembled MEMS ES-VEH structure: (a) individual components and (b) overview of assembled structure.

\section{Typical Harvesting Experiments and Results}

The main experiments involved driving and measuring the dynamic motion and electrical vibration of the harvested voltage using a combination of a laser Doppler vibrometer, a shaker, an amplifier, and an accelerometer. The measured oscillation was captured using several harvested voltages from $\mathrm{CE}_{1}$ and $\mathrm{CE}_{2}$. An overview of the experimental setup is shown in Fig. 4(a). The experimental apparatus consisted of a laser to capture the dynamic motion and a shaker to generate the in-plane motion. For practical oscillation measurements, a pick-up accelerometer was applied. The electrical oscillation was $0.2 \mathrm{mg}$-p at $40 \mathrm{~Hz}$, the electrical load $R_{L}$ was $1 \mathrm{M} \Omega$, and the capacitance of the probe for the oscilloscope was $32 \mathrm{pF}$, as shown in Fig. 4(b). The measured relative motion was $50 \mu \mathrm{m}$ and the voltage was $0.1 \mathrm{~V}$. The harvested voltage was small when the relative motion was large because $R_{L}$ was much smaller than the optimum impedance. To evaluate the surface potential, the acceleration and frequency were kept the same in the following experiments.

\section{Experimental Evaluation of Surface Potential by Proposed Method}

After setting up the experimental apparatus, a bias voltage was applied to evaluate the negative surface potential. The bias voltage was set at $V_{b s}$ located at the bottom of $V_{c n}$, as shown in Fig. 5. $\mathrm{CE}_{2}$ was connected to the ground to cancel its voltage. The load resistance $R_{L}$ was connected to $\mathrm{CE}_{1}$ to measure its voltage. The average RMS calculated using the measured voltage oscillation at the time domain is shown in Fig. 6. The lowest measured voltage was around $130 \mathrm{~V}$. It is assumed that the external bias was balanced to the potential of the electret; thus, the measured voltage was smallest at small electrical biases for the variable capacitors in ES-VEH. The voltage curve shows the shape of a valley. In contrast to the negative bias response that indicates a large electrical bias of the capacitor, the voltage curve 


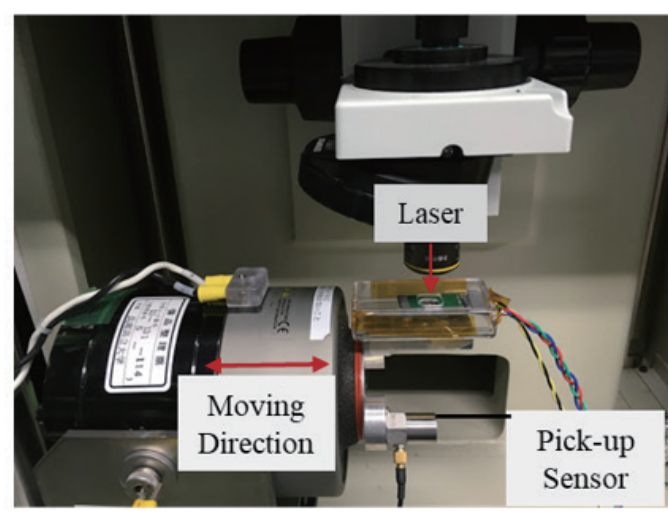

(a)

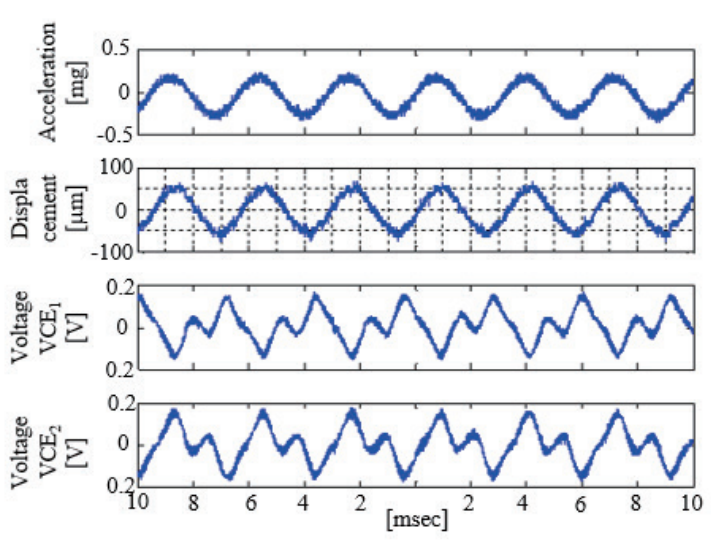

(b)

Fig. 4. (Color online) (a) Overview of experimental setup and (b) time-scale oscillations (acceleration, displacement, and harvested voltage).

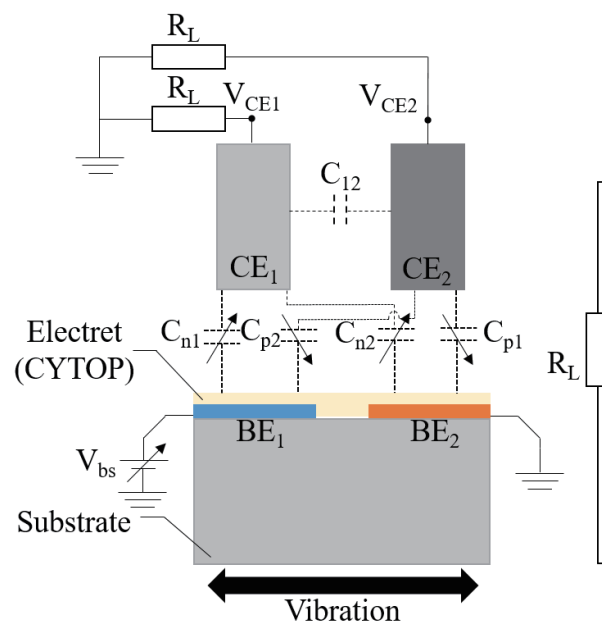

(a)

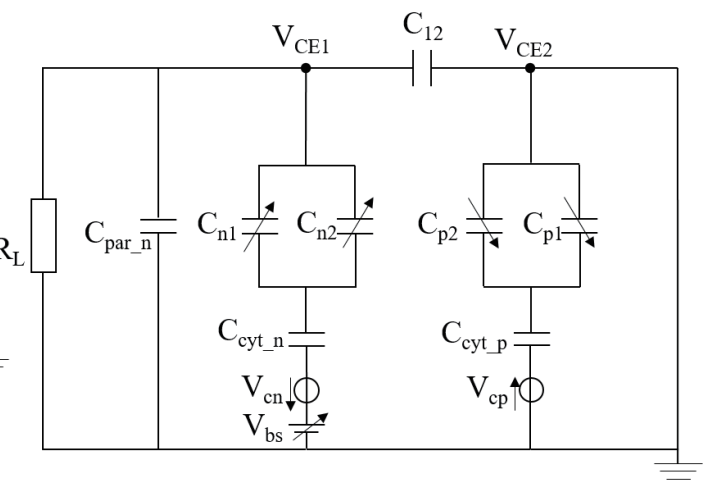

(b)

Fig. 5. (Color online) Experimental evaluation of surface potential: (a) schematic diagram of proposed method and (b) equivalent circuit.

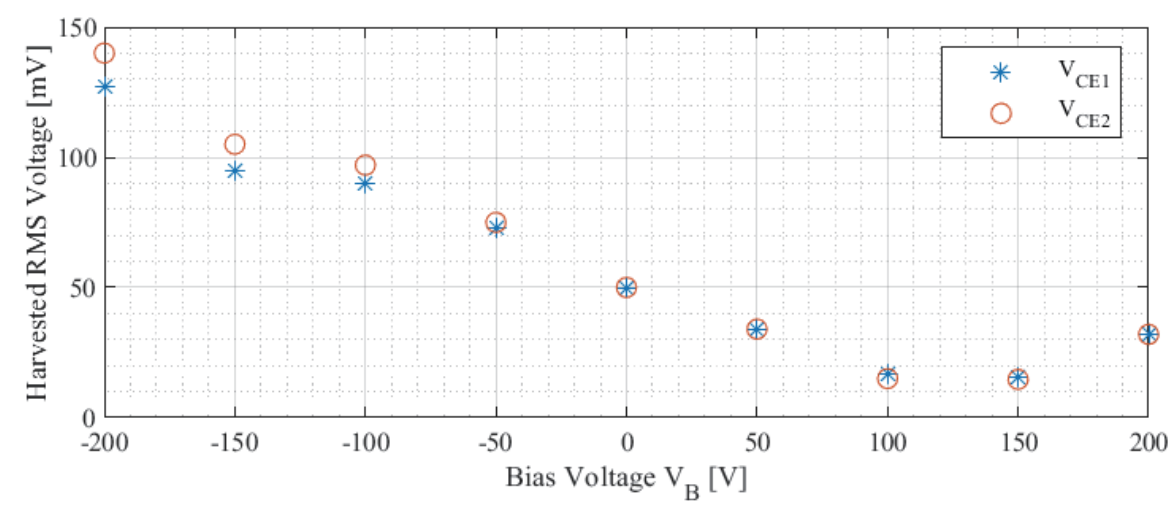

Fig. 6. (Color online) RMS voltages of harvested power. 
increases with the applied voltage. The responses to the applied external biases indicate that the biases controlled the electrical potential in the electret. At the smallest voltage captured, the variable capacitors did not include sufficient voltage for harvesting. These results show that the proposed method can be used to evaluate the practical surface potential in the electret from variable harvested voltages with external biases.

\section{Discussion}

Evaluation results of the surface potential are described above. When external biases were applied in the positive DC direction, the measured voltage curve showed a valley shape with a minimum point of around $130 \mathrm{~V}$, which is smaller than the surface potential of $150 \mathrm{~V}$. The practical ES-VEH is evaluated after charging, which took a long time, so the actual voltage decreased because the charged ions were excluded.

\section{Conclusions}

In this study, the surface potential of the MEMS ES-VEH was evaluated by the biasing method. The experimental technique was proposed and a complete prototype was fabricated by silicon-MEMS processing. We conclude that it is possible to experimentally evaluate the surface potential of bipolar charging by using an external voltage approach.

\section{Acknowledgments}

This work was partially supported by the Japan Society for the Promotion of Science (JSPS) KAKENHI Grant-in-Aid for Scientific Research (C) Number 19K04533 for the scientific work in the University of Hyogo.

\section{References}

1 M. Ben-Daya, E. Hassini, and Z. Bahroun: Int. J. Prod. Res. 57 (2019) 4719. https://doi.org/10.1080/00207543.2 017.1402140 .

2 F. Shrouf, J. Ordieres, and G. Miragliotta: 2014 IEEE Int. Conf. Industrial Engineering and Engineering Management 2014 (2014) 697-701. https://doi.org/10.1109/IEEM.2014.7058728.

3 D. Zuehlke: Control 34 (2010) 129. https://doi.org/10.1016/j.arcontrol.2010.02.008.

4 Y. Liao, F. Deschamps, E. de F. R. Loures, and L. F. P. Ramos: Int. J. Prod. Res. 55 (2017) 3609. https://doi.org/ 10.1080/00207543.2017.1308576.

5 W. K. G. Seah, Z. A. Eu, and H.-P. Tan: 2009 1st Int. Conf. Wireless Communication, Vehicular Technology, Information Theory and Aerospace Electronic Systems Technology (2009) 1-5. https://doi.org/10.1109/ WIRELESSVITAE.2009.5172411.

6 Y. Wu, A. Badel, F. Formosa, and W. Liu: J. Intell. Mater. Syst. Struct. 24 (2012) 1445. https://doi. org/10.1177/1045389X12470307.

7 I. Kanno: J. Phys. Conf. Ser. 660 (2015) 012001. https://doi.org/10.1088/1742-6596/660/1/012001.

8 Y. Tan, Y. Dong, and X. Wang: J. Microelectromech. Syst. 26 (2017) 1. https://doi.org/10.1109/ JMEMS.2016.2611677.

9 Y. Suzuki: IEEJ Trans. Electr. Electron. Eng. 6 (2011) 101. https://doi.org/10.1002/tee.20631.

10 K. Yamamoto, A. Badel, F. Formosa, L. Charleux, T. Fujita, K. Kanda, and K. Maenaka: J. Phys. Conf. Ser. 1052 (2018) 012122. https://doi.org/10.1088/1742-6596/1052/1/012122. 
11 K. Minami, N. Miwatani, K. Kanda, T. Fujita, and K. Maenaka: J. Phys. Conf. Ser. 660 (2015) 012107. https:// doi.org/10.1088/1742-6596/660/1/012107.

12 S. Boisseau. G. Despesse, and A. Sylvestre: Smart Mater. Struct. 19 (2010) 075015. https://doi. org/10.1088/0964-1726/19/7/075015.

13 B. Vysotskyi, D. Aubry, P. Gaucher, X. Le Roux, F.Parrain, and E. Lefeuvre: J. Micromech. Microeng. 28 (2018) 074004. https://doi.org/10.1088/1361-6439/aabc90.

14 M. Edamoto, Y. Suzuki, N. Kasagi, K. Kashiwagi, Y. Morizawa, T. Yokoyama, T. Seki, and M. Oba: 2009 IEEE 22nd Int. Conf. Micro Electro Mechanical Systems (2009) 1059-1062. https://doi.org/10.1109/ MEMSYS.2009.4805569.

15 P. Basset, D. Galayko, A. M.Paracha, F. Marty, A. Dudka, and T. Bourouina: J. Micromech. Microeng. 19 (2009) 115025. https://doi.org/10.1088/0960-1317/19/11/115025.

16 T. Fujita, T. Toyonaga, K. Nakade, K. Kanda, K. Higuchi, and K. Maenaka: Proc. Eng. 5 (2010) 774. https:// doi.org/10.1016/j.proeng.2010.09.223.

17 V. Leonov, R. van Schaijk, and C. Van Hoof: IEEE Sens. J. 13 (2013) 3369. https://doi.org/10.1109/ JSEN.2013.2263636.

18 K. Minami, T. Fujita, T. Onishi, K. Sonoda, N. Miwatani, K. Kanda, and K. Maenaka: IEEJ Trans. Electr. Electron. Eng. 135 (2015) 116. https://doi.org/10.1541/ieejsmas.135.116.

19 K. Sonoda and T. Onishi: IEEJ Trans. Electr. Electron. Eng. 135 (2015) 91. https://doi.org/10.1541/ ieejsmas.135.91.

20 Y. Suzuki, D. Miki, M. Edamoto, and M. Honzumi: J. Micromech. Microeng. 20 (2010) 104002. https://doi. org/10.1088/0960-1317/20/10/104002.

\section{About the Authors}

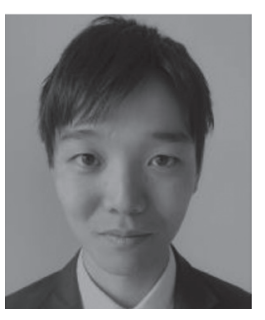

Koki Yamamoto received his B.E. and M.E. degrees from the University of Hyogo, Japan, in 2014 and 2016, respectively. He received his Ph.D. degree from École doctorale SISEO of Université Grenoble Alpes, Grenoble, France, in 2019. From 2016 to 2019, he worked as a Contrat doctoral in SYMME Laboratory, Université Savoie Mont Blanc, Annecy, France. Since 2019, he has been a researcher of the Mechanical Elements, Vibration and Acoustic Section of Ebara Corporation, Kanagawa, Japan. His research interests are in MEMS, energy harvesting, and nonlinear dynamical systems. (yamamoto.koki@ebara.com)

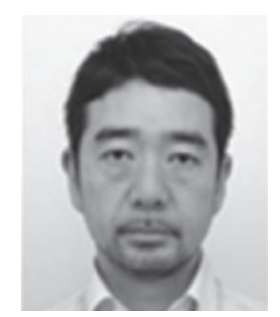

Takayuki Fujita received his B.E., M.E., and Ph.D. degrees from the Himeji Institute of Technology, Japan in 1995, 1997, and 2000, respectively. From 2001 to 2007, he was a research associate at the Himeji Institute of Technology. From 2008 to 2013, he was the Power group leader of the Maenaka Human-Sensing Fusion Project supported by the Japan Science and Technology Agency. From 2013 to 2014, he worked as a visiting researcher at the IMEC Holst Centre, the Netherlands. He is currently an associate professor in the Department of Electronics and Computer Science, University of Hyogo. His research interests are in MEMS and vibration energy harvesters. (fujita@eng.u-hyogo.ac.jp) 


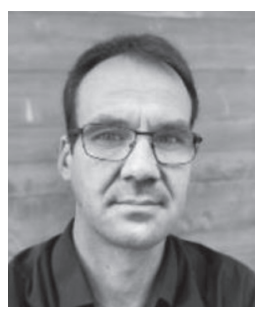

Adrien Badel received his M.S. degree in electrical engineering from Institute National des Sciences Appliquées de Lyon (INSA), Lyon, France in 2002, and his Ph.D. degree in 2005 while working at the Electrical Engineering and Ferroelectricity Laboratory of INSA Lyon. He received his Ph.D. degree on vibration control and energy harvesting. From November 2005 to November 2007, he was a Japan Society for the Promotion of Science (JSPS) postdoctoral fellow at the Institute of Fluid Science of Tohoku University, Sendai, Japan. He is now a professor at the Laboratory of Systems and Materials for Mechatronics of the Université Savoie Mont Blanc, Annecy, France. His research interests include energy harvesting, vibration damping, and piezoelectric actuator modeling and control. (adrien.badel@univ-smb.fr)

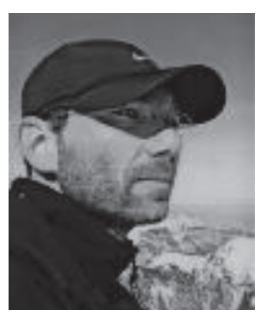

Fabien Formosa received his M.S. degree from the École Normale Supérieure de Cachan, Cachan, France, in 1999, and his Ph.D. degree from Université Paris VI in 2002. He is currently an associate professor at Université Savoie Mont Blanc, Annecy, France. His expertise is on the design of miniature systems for energy conversion, such as microheat engines (microStirling) and vibration energy harvesting. His activities range from the analytical modeling of multiphysics systems to the technological integration of smallscale devices. (fabien.formosa@univ-smb.fr)

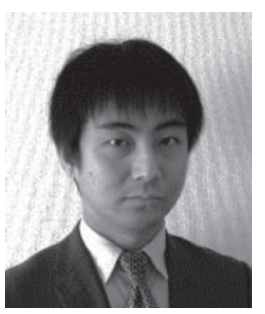

Kensuke Kanda received his B.E., M.E., and Ph.D. degrees in mechanical engineering from Tokyo Metropolitan University, Japan in 2000, 2003, and 2006, respectively. From 2006 to 2008, he worked for the Advanced Software Technology and Mechatronics Research Institute of Kyoto, Japan. From 2008 to 2010, he worked for the Maenaka Human-Sensing Fusion Project of JST. Since 2010, he has been an assistant professor at the University of Hyogo. His research interests are in microfabrication technology, piezoelectric microdevices (piezo-MEMS), power harvesters, and their integration. (kanda@eng.u-hyogo.ac.jp)

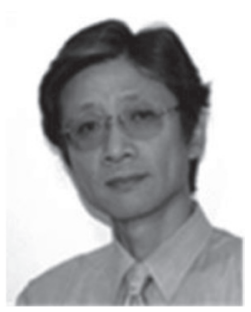

Kazusuke Maenaka received his B.E., M.E., and Ph.D. degrees from Toyohashi University of Technology, Japan, in 1982, 1984, and 1990, respectively. Since 1993, he has been with the Department of Electronics of Himeji Institute of Technology. After the unification of the universities in Hyogo Prefecture in April 2004, he joined the University of Hyogo as a professor. From 2008 to 2013, he was the project leader of the Maenaka Human-Sensing Fusion Project supported by the Japan Science and Technology Agency. His research interests are in MEMS devices and technology, particularly silicon mechanical sensors, and their integration. (maenaka@eng.u-hyogo.ac.jp) 